$$
\text { CONF- } 9510263 \cdots-13
$$

\title{
INDUCTION-ACCELERATOR HEAVY-ION FUSION: STATUS AND BEAM PHYSICS ISSUES
}

\author{
Alex Friedman \\ Lawrence Livermore National Laboratory, University of California \\ P.O.Box 5508, L-440, Livermore, California 94550 USA WUCDY \\ JUN 059996 \\ OSTI
}

This paper was prepared for submittal to the Proceedings of the 8th ICFA Advanced Beam Dynamics Workshop on Space Charge Dominated Beams and Applications of High Beam Brightness Beams, Bloomington, IN, October 10-13, 1995

January 26, 1996

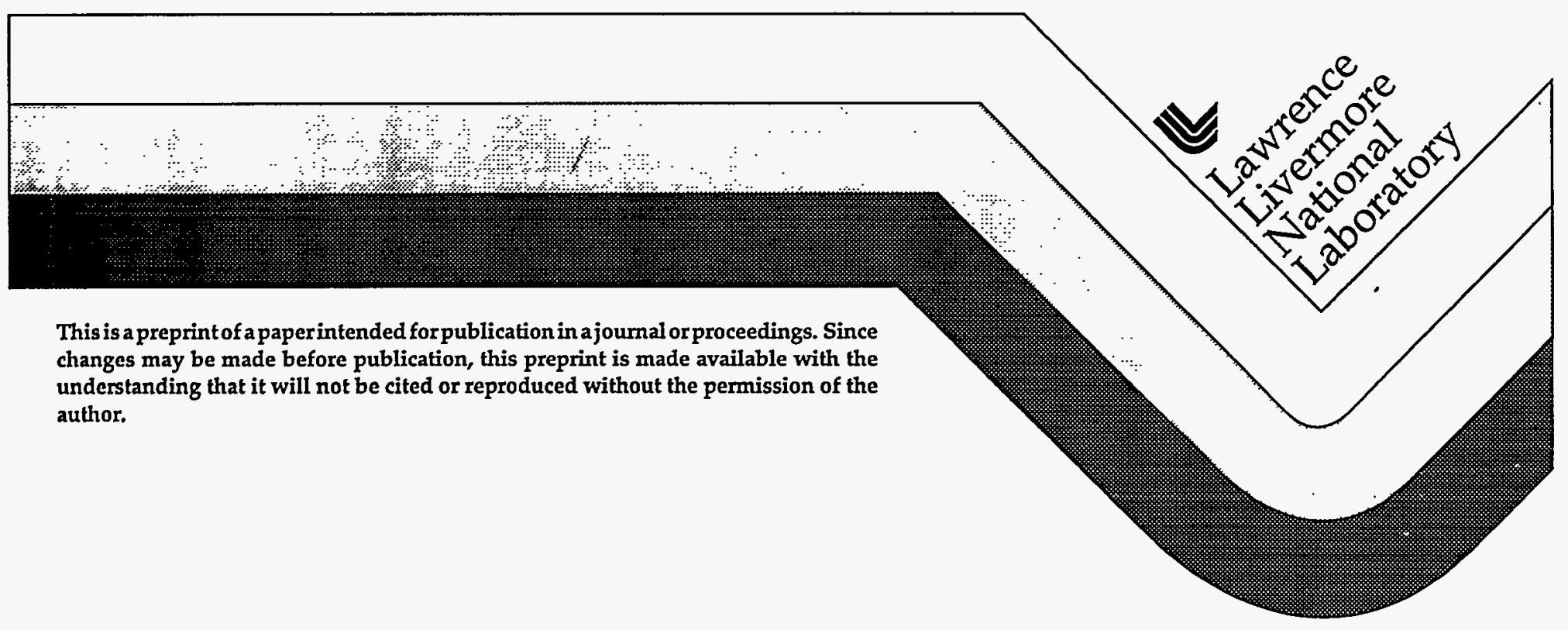




\section{DISCLAIMER}

This document was prepared as an account of worksponsored by an agency of the United States Government. Neither the United States Govemment nor the University of California nor any of their employees, makes any warranty, express or implied, or assumes any legal liability of responsibility for the accuracy, completeness, or usefulness of any information, apparatus, product, or process disclosed, or represents that its use would not infringe privately owned rights. Reference herein to any specific commercial products, process, or service by trade name, trademark, manufacturer, or otherwise, does not necessarily constitute or imply its endorsement, recommendation, or favoring by the United States Government or the University of Caiifornia. The views and opinions of authors expressed herein do not necessarily state or reflect those of the United States Government or the University of California, and shall not be used for advertising or product endorsement purposes. 


\title{
INDUCTION-ACCELERATOR HEAVY-ION FUSION: STATUS AND BEAM PHYSICS ISSUES*
}

\author{
Alex Friedman \\ Lawrence Livermore National Laboratory \\ University of California \\ Livermore, CA 94550 USA
}

\begin{abstract}
Inertial confinement fusion driven by beams of heavy ions is an attractive route to controlled fusion. In the U.S., induction accelerators are being developed as "drivers" for this process. This paper is divided into two main sections. In the first section, the concept of induction-accelerator driven heavy-ion fusion is briefly reviewed, and the U.S. program of experiments and theoretical investigations is described. In the second, a "taxonomy" of space-charge-dominated beam physics issues is presented, accompanied by a brief discussion of each area.
\end{abstract}

\section{INDUCTION-ACCELERATOR HIF CONCEPT AND STATUS}

\section{Overview}

The U.S. is developing the physics and technology of ion induction accelerators, with the goal of electric power production by means of heavy-ion beam-driven inertial fusion (heavy-ion fusion, or HIF). In addition, heavy-ion induction accelerators are an attractive driver option for a high-yield microfusion facility for defense research.

Accelerators for high-energy physics research typically have long lifetimes and good availability; an accelerator designed as a fusion driver should share these attributes. Since the beams will be focused onto the target by magnetic fields, the final lens will not be subject to serious damage from the exploding targets. Induction accelerators in particular are efficient, low-impedance devices that naturally accelerate a high-current beam, can readily amplify the current of that beam, and can easily meet repetition rate requirements of a few pulses per second. For these reasons, the heavy-ion induction accelerator is the driver being pursued for an inertial confinement fusion (ICF) power plant in the U.S. Inertial Fusion Energy (IFE) program, in the Office of Fusion Energy of the U.S. Department of Energy. However, significant technology development must be carried out before an HIF driver is realized. Because driver development is deemed the long lead-time item in the IFE development sequence, much of the programmatic emphasis is currently being placed on high-current ion accelerator research.

For a fusion power technology to be viable, it must offer an economically attractive power plant at a reasonable size (of order $1 \mathrm{GW}$ electric output, a smaller plant size being preferable), and must have favorable properties with respect to environmental considerations. Recent studies indicate that the cost of electricity from an HIF power plant can compare favorably with those of tokamak fusion, future fission and coal, and suggest that the environmental attributes can be benign as well. $[1,2,3]$ 
Furthermore, the development program leading to a pilot power plant must be affordable. The HIF development cost will be minimal because the program builds upon a multi-billion dollar worldwide investment in particle accelerators, and upon a large and multi-faceted inertial fusion program based upon lasers and light-ion drivers. The planned National Ignition Facility (NIF), to be based upon a 1.8 MJ glass laser, can test the most important heavy-ion target physics issues: soft $\mathrm{X}$-ray transport and drive symmetry, hohlraum plasma dynamics, capsule implosion hydrodynamics, and mixing of unwanted material into the central fuel. It will be possible to deploy experiments on the NIF which focus the laser beams into smaller hohlraums (filled with high-atomic-number doped gas at 1/10 the critical density) placed at each end of the larger hohlraum which contains the fusion capsule. These will emit their $\mathrm{X}$-rays into the main hohlraum in the same way as the converters at the ends of a two-sided heavy-ion fusion hohlraum. Experiments using light-ion drivers will also yield information which is important to heavy-ion scenarios, including ion energy deposition and conversion to $\mathrm{x}$-rays in foams, radiation transport, capsule symmetry requirements, and beam propagation in the target chamber.

The use of a non-solid first surface in the chamber obviates any need for a separate large-scale materials testing facility; experiments on the NIF and other lasers will provide much of the needed data. An Engineering Test Facility (ETF) accelerator built to explore target physics and target-chamber dynamics can be used in the subsequent demonstration power plant as well. In one scenario, such a program might be pursued as an international effort. In another scenario, the ETF driver might also serve as the driver for a high-yield microfusion capability for defense research.

These multiple-use attributes are made possible by the physical decoupling of the driver, the fusion chamber, and the target. A beam switchyard will enable one accelerator to drive several target chambers at the same time. The cost penalty associated with the requisite pulse repetition rate should be negligible. Reducedyield targets can be used in scaled fusion chambers, enabling the resolution of nuclear engineering issues at minimal cost. Finally, an accelerator driver can be built in stages, thereby combining the accelerator development program with the construction of the eventuál facility.

Research into HIF drivers outside the U.S. has emphasized radio-frequency (RF) linac technology in conjunction with storage rings. The U.S. induction-based research program and the RF-based efforts are complementary. The decision to pursue induction technology in the U.S. was made for well-defined reasons, including a lower predicted driver cost, and a belief that the critical issues arise at lower energy and so can be more readily resolved. RF linac technology is mature, but the required storage rings are challenging; the approach may prove entirely adequate to the fusion-driver task. Discussions of the advantages of each of these approaches can be found in Refs. [4,5].

In Subsection 2 below, we review the basic design of a radiatively-driven heavy-ion target using "two-sided" illumination (with beams in two clusters), and the requirements imposed by such a target upon the incoming beams.

In Subsection 3 we present a schematic of an induction linear accelerator designed around these requirements. The research program is working toward 
expansion of the usable design regime of a linear accelerator, toward a lower final particle energy. These considerations are also discussed in that subsection.

The recirculating induction accelerator promises cost reduction by re-using the same accelerating and focusing (confining) elements multiple times.[6] The recirculator concept is discussed in Subsection 4.

The goals and design of the Elise accelerator are briefly described in Subsection 5.[7] Proposed for construction at Lawrence Berkeley National Laboratory (LBNL), Elise is an outgrowth of the ILSE (Induction Linac Systems Experiments) concept, and represents the initial phase of that overall program.[8] Elise was designed to be a $5 \mathrm{MeV}$, multi-beam induction linac accelerating potassium ( $\mathrm{K}^{+}$, 39 AMU) ions produced by LBNL's new electrostatic-quadrupole (ESQ) injector, which is also described briefly in Subsection 5. The ILSE accelerator is intended to serve as a testbed upon which a variety of experiments using driver-scale (in diameter and line-charge density) beams will be carried out. These will ultimately include beam merging, drift-compression, and focusing onto a small spot. The Elise project is currently "on hold."

A recently initiated experimental program in beam bending and recirculation at LLNL is described in Subsection 6.[9] The small prototype recirculator which is the ultimate goal of this work will be the first accelerator of its type. Because of the unique opportunity they will afford for the study of space-charge-dominated beam behavior on a long time scale, experiments on the small recirculator will be important to linear accelerators as well.

Finally, ongoing research is examining issues including beam propagation in the target chamber, long-time beam dynamics, and detailed beam optics in structures including injectors, beam combiners, plasma lenses, and magnetically confined transport of space-charge-dominated beams. A brief outline of these elements of the program is presented in Subsection 7.

\section{Review of Requirements Imposed by the Target}

The U.S. HIF program is examining a variety of target concepts. In one illumination geometry, the fusion capsule is enclosed in a hohlraum, which has a radiation converter of radius $\sim 2-6 \mathrm{~mm}$ at each end. Each converter is illuminated by a cluster of beams with a narrow cone angle. This geometry is similar enough to that of an indirectly-driven laser fusion target that the design methods and tools used for the latter carry over directly. Furthermore, the illumination geometry is favorable for power-plant design, relative to concepts.which require that the beams enter the fusion chamber from a wide multiplicity of directions. To achieve a gain of 10-100, such a target is projected to require a total incident beam energy of order $5 \mathrm{MJ}$ in a suitably shaped pulse, with duration (for the main part of the pulse) of order $10 \mathrm{~ns}$ and a longer initial "foot" preceding. Other geometries include spherical or nearly-spherical targets, two-sided targets with the converter material distributed appropriately around the inside of the main hohlraum, single-sided targets with a single radiator, and "single-sided" targets with two radiators which are illuminated "sideways" (at $90^{\circ}$ to the symmetry axis of the main hohlraum) by beam clusters coming from ports on the same side of the fusion chamber. 


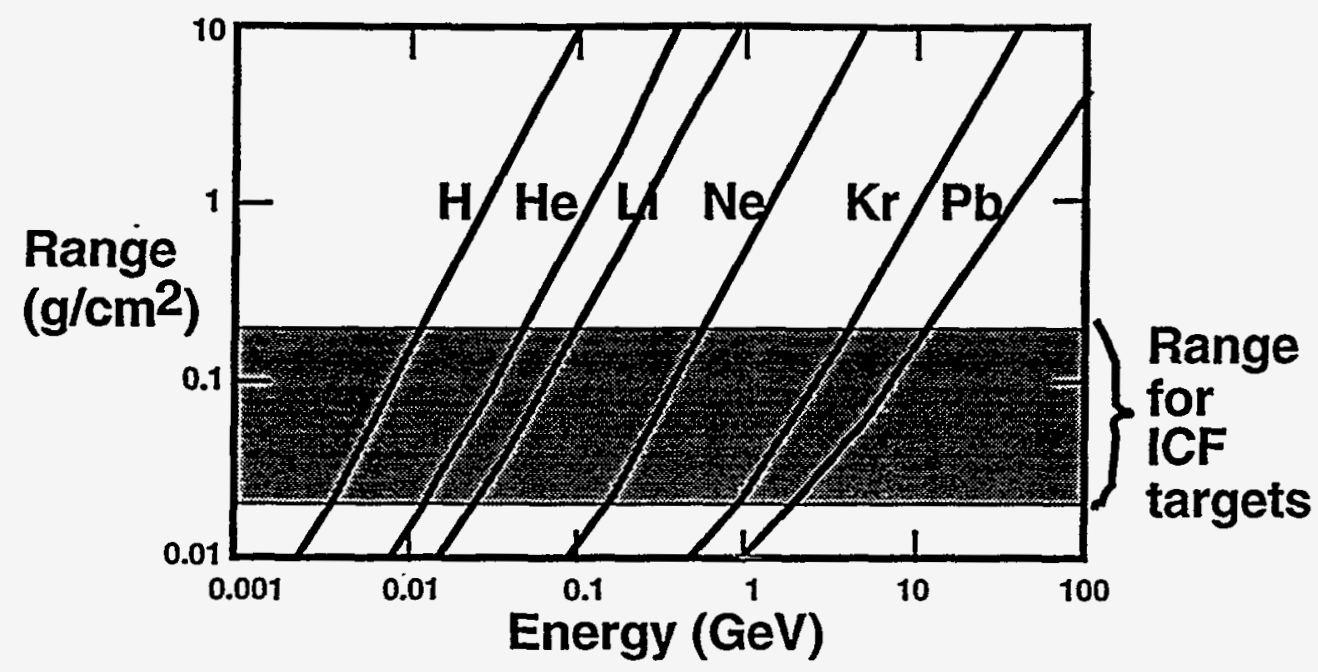

Fig. 1. Range-energy relation for various ions stopping in matter.

The energy-range relation of ions stopping in matter (Fig. 1) is an important factor in selecting the ion energy. It is necessary to stop the incident ions in a relatively small amount of matter (range $R \sim 0.02-0.2 \mathrm{~g} / \mathrm{cm}^{2}$ ) if the amount of converter material heated by the incident beams is to be kept small enough for a specific energy deposition of order $10^{8} \mathrm{~J} / \mathrm{g}$; the energy devoted to overcoming the specific heat of the converter material must be minimized if high conversion efficiency of ion energy to x-rays is to be obtained. For an ion of mass $~ 100$ $200 \mathrm{AMU}$, the required energy per ion is found to be of order $1-10 \mathrm{GeV}$. For a light ion of mass -1-7 AMU, the corresponding energy is of order 3-100 MeV. The heavy-ion fusion program has emphasized drivers using singly-charged mass 100-200 ions because their relatively high allowed energy allows the requisite $\sim 5 \mathrm{MJ}$ to be achieved at a relatively low current (compared to that of a lighter ion scenario), which in turn allows non-neutralized ballistic focusing of the beam onto the target in near-vacuum. The achievable power on target is limited by several effects which add roughly in quadrature; these include beam space charge, beam emittance (thermal presșure), misalignments, and aberrations. The dependence of the focusable power upon ion energy as set by space charge is especially steep, varying as the $5 / 2$ power.[5] Thus for non-neutralized focusing there is a strong incentive to operate at high ion energy and correspondingly large ion mass. However, as discussed in the next section, use of a lighter ion, or a higher charge state, may convey certain advantages, despite a requirement of at least partially neutralized transport to the target.

Most power plant concepts call for near-ballistic focus of the beams through a fusion chamber with radius $4-10 \mathrm{~m}$. If one assumes a $10 \mathrm{~m}$ separation between the middle of the final magnetic optic and the target, and a $3 \mathrm{~mm}$ radius spot on target, the particles must be aimed with a "microdivergence" angle of $0.003 / 10=3 \times 10^{-4}$ radians. This same angle represents the ratio of transverse thermal velocity to directed beam velocity, and its square $\left(\sim 10^{-7}\right)$ represents the ratio of transverse thermal to directed energies. For a $10 \mathrm{GeV}$ beam, the transverse temperature must 
remain below $\sim 1 \mathrm{keV}$. The transverse emittance, or projected phase space area, is the product of beam transverse dimension and velocity spread, the latter normalized to the directed velocity and so measured as an angle. The final optic does not produce a perfect image; geometric and chromatic aberrations limit the achievable spot size. Uncorrected optics are limited to a focusing angle of about $15 \mathrm{mrad}$. For a beam with a focal spot radius of $3 \mathrm{~mm}$ focused through a $15 \mathrm{mrad}$ angle from a maximum radius $15 \mathrm{~cm}$, one obtains an emittance requirement of $\sim \pi(150 \mathrm{~mm})(0.3 \mathrm{mrad})=\pi(3 \mathrm{~mm})(15 \mathrm{mrad})=45 \pi$ - $\mathrm{mm}$-mrad (ignoring space charge). In addition, space charge causes beam spreading near the target, and the beam centroid must be precisely aimed and must not wander more than about $0.2 \mathrm{~mm}$. These effects contribute jointly to the effective spot size; to compensate, the emittance must be somewhat smaller than that calculated above.

Similarly, the ability of the optical system to focus ions with a range of energies onto a small spot is limited. Chromatic aberrations limit the relative momentum variation $\delta \mathrm{p}_{z} / \mathrm{p}_{\mathrm{z}}$ to about $0.3 \%$. Optically-corrected focusing designs are employed in other accelerator applications, and have been studied for HIF [10], but remain to be shown to be practical.

Alternative modes of transporting the beams to the target include neutralized or partially neutralized ballistic transport, as well as self-pinched propagation in a channel. Neutralization may be important if operation at higher chamber pressures associated with a greater shot-to-shot repetition rate (for some chamber designs) is to be practical. By easing the effects of space charge, these modes open up a larger region in parameter space at lower ion energy and mass. Detailed simulation studies have shown that, for the nominal HYLIFE-II parameters, [1] even a relatively small degree (perhaps half a percent) of ionization of the background chamber vapor can result in excellent beam neutralization, and this nearly neutralized ballistic regime is well on its way toward replacing transport in near vacuum as the baseline model.[11,12] Pinch-mode propagation would offer a number of advantages, including smaller holes in the chamber wall, and acceptance of beams with a greater longitudinal velocity spread.[13] Recent progress in plasma lenses suggests that they may be useful in the introduction of a beam into such a channel.[14] However, these latter regimes remain less well understood.

\section{Linear Accelerator Concepts}

Induction accelerators apply electromotive force to the beams by passing them through a series of one-turn pulsed transformers (see Fig. 2). Pulsed power is routed to the transformer's "primary winding;" conducting surfaces are arranged so that an axial EMF appears across an "accelerating gap" in the beam pipe. The ferromagnetic core, which has a large permeability, gradually fills with magnetic flux. A short-circuit across the input is averted until the core's "Volt-seconds" have been consumed, affording almost-d.c., relatively flat pulses. Longitudinal confinement of the beams requires voltage "ears," or temporal ramps on the ends of the voltage waveform timed to coincide with the passage of the front and back tips of the beams through the accelerating gap (see Fig. 3). In contrast with RF accelerators, there is no requirement for constant frequency, so longitudinal 


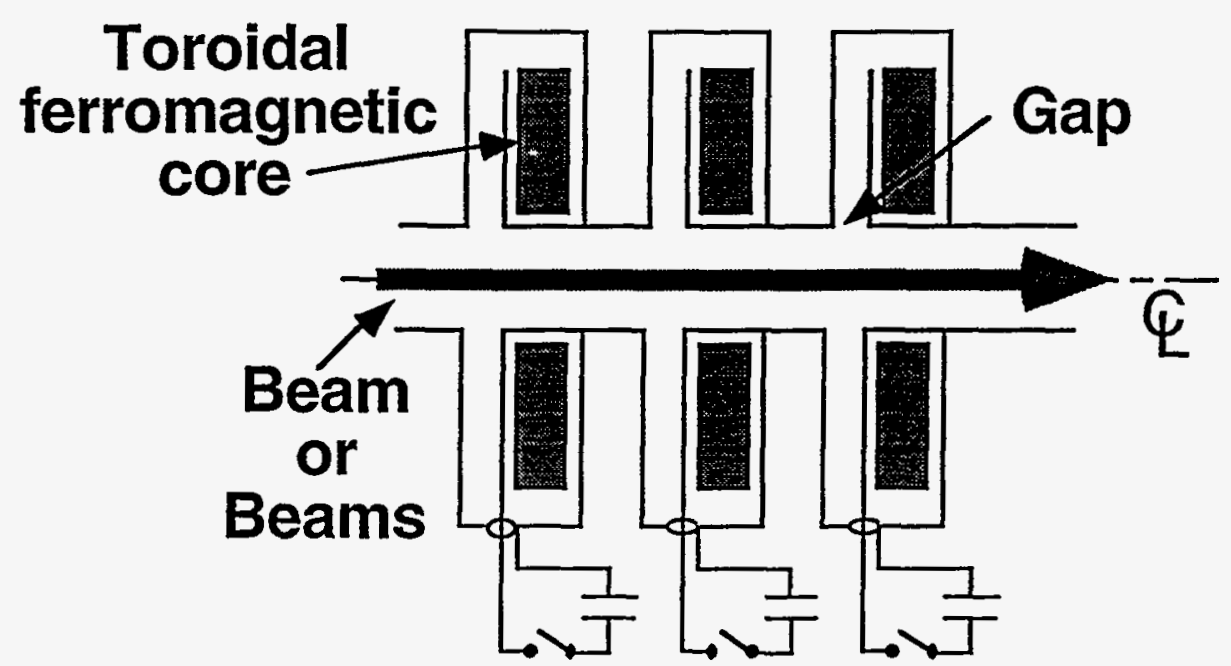

Fig. 2. Induction module geometry.

compression and increasing velocity can produce an increasing current, up to the limits of the transverse confining fields.

A "conventional" linear induction accelerator designed using ions of mass $\sim 200$ to meet the requirements imposed by the "conventional" target is depicted schematically in Fig. 4. Such an accelerator achieves economy by passing multiple beams through each induction core. Power amplification to the required 10 $14-10^{15}$ $\mathrm{W}$ is achieved by beam combining, acceleration, and longitudinal bunching. The accelerator lattice must transversely "focus" the beam (confine it against its own space charge, and to a much lesser degree its own thermal pressure); in modern accelerators this is effected with alternating-gradient quadrupole lenses, which may be either electrostatic or magnetic. Each lens focuses the beam in one plane while defocusing it in the other; however, because the beam is larger (and thus experiences stronger fields) as it passes through a focusing element, the net effect is a strong focusing.

In such a "conventional" HIF accelerator, multi-beam injection with 16-64 beamlets is followed by acceleration with electrostatic transverse focusing. Fourinto-one beam merging, is effected at $10-100 \mathrm{MeV}$, with a transition to magnetic

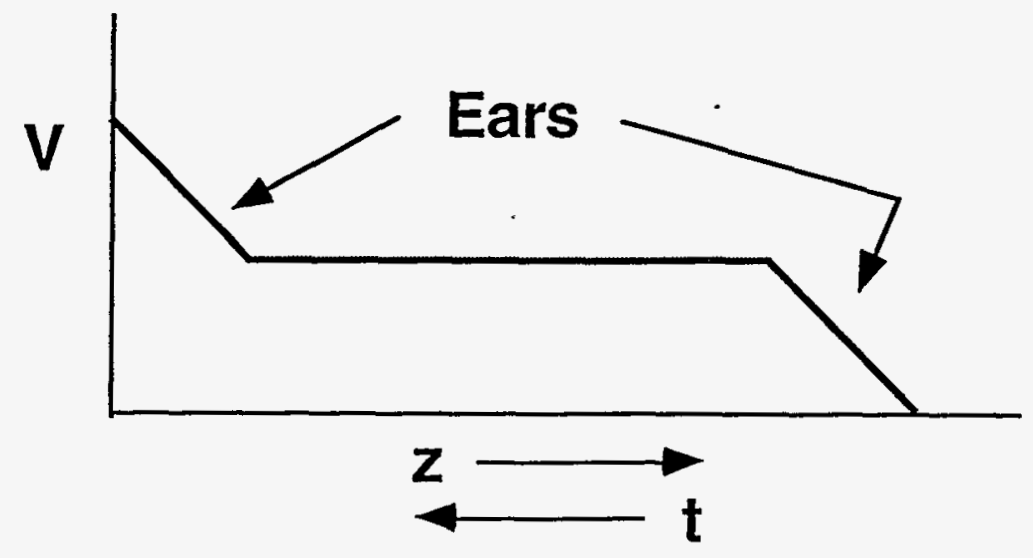

Fig. 3. Typical accelerating waveform. 


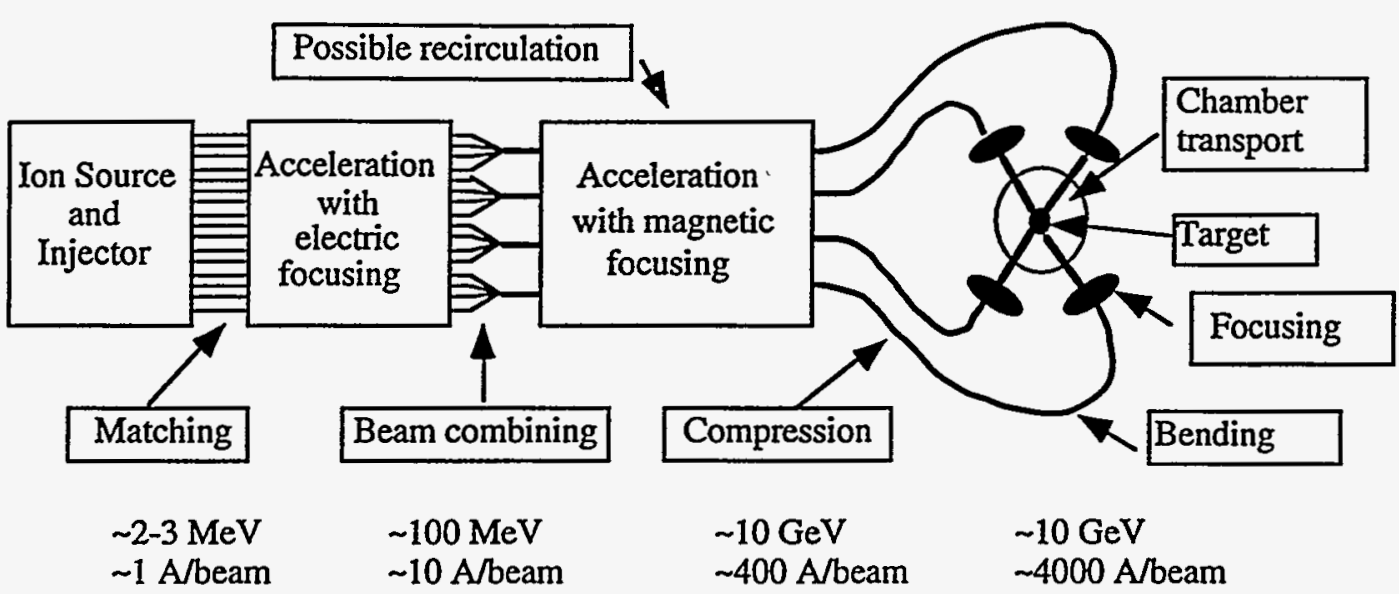

Fig. 4. Schematic of a "conventional" induction accelerator driver.

focusing in roughly the same energy range. The linear approach, in comparison with the recirculating approach discussed below, enjoys relatively simple timing and control, acceleration and focusing tailored to the beam at each point, no need for bends except near the target, and reduced vacuum requirements because debris from the wall is readily pumped away between shots and does not accumulate. However, the length and need for a high gradient $\sim 1 \mathrm{MV} / \mathrm{m}$ establish the cost.

In present designs the major part of the accelerator employs magnetic focusing. It may well be possible to accelerate beams in an induction linac with an average gradient exceeding $1 \mathrm{MV} / \mathrm{m}$, by suitable grading of insulators. The cost penalty of the larger cores needed for a higher gradient would be more than offset by the reduced length.

Beam merging[15] is likely to be an important cost-reduction technique because at low energy it is desirable to employ many beams, while at high energy economics drives the design toward fewer beams. Electrostatic quadrupoles are generally to be preferred at low energy because their focusing power scales more favorably with velocity at low energy. Also at low energy the quadrupoles must be closely spaced along the axis to provide sufficient confinement, and it is difficult to fit electromagnets in. Finally, electrostatic elements are cheaper to fabricate. The limits imposed by voltáge breakdown imply that a small aperture, and thus a relatively large number of beams, must be used. In contrast, at higher energy focusing should use magnetic quadrupoles. Longitudinal compression at constant radius is possible with magnets because the confinable line charge density rises with the beam energy. Also, if small-aperture magnets were used, most of the induction core's bore would be filled with superconducting magnet insulation and coils instead of beam. Thus, at high energy, efficient core use dictates a large aperture, and a smaller number of thicker beams.

However, beam merging inevitably leads to an emittance increase because empty phase space is entrained in the combining process. This can be minimized by bringing the beams together in a "Stonehenge" geometry, which gives good packing. Such a geometry is depicted in Fig. 5 .

As explained above, a high ion mass (of order 200) allows rigid beams with high energy, of order $10 \mathrm{GeV}$, and implies that relatively few ions are needed, 


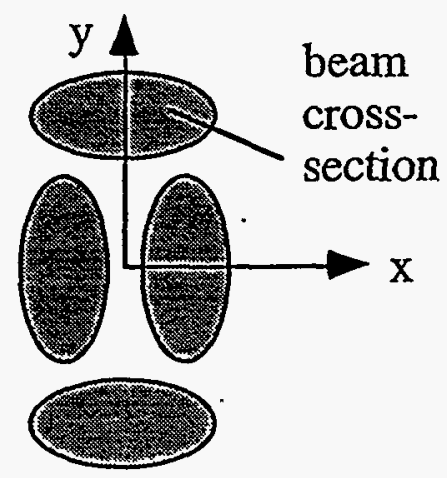

Fig. 5. "Stonehenge" geometry

reducing collective effects and easing focusing requirements. The baseline induction linac concept assumes a kinetic energy $\sim 10 \mathrm{GeV}$. At lower masses, preservation of a small ion range in the target implies that the ion energy must be reduced. Use of a reduced ion energy would permit a shorter accelerator, and a smaller ion stopping range would enhance the target gain. However, the required current to produce $\sim 5 \mathrm{MJ}$ on target is considerably higher, and so a detailed optimization must be carried out. Smaller ion masses (as low as 39) are routinely used in present-day and near-term experiments. LBNL, LLNL, and Sandia National Laboratories are working together to understand these tradeoffs as they apply to the various accelerator technologies being considered for ion-beam driven fusion.

Substantial improvements in cost and efficiency of induction linacs may well be possible through the use of lower-energy ions. The energy gain in a core is given by the product $I V \tau$, where $I$ is the beam current, $V$ the voltage gain, and $\tau$ the pulse duration. Because cost and losses increase with increasing $V \tau$, a large current and small voltage would be preferable. The minimum beam energy is set by the scaling of focusable current onto the target (assuming unneutralized focusing), with the beam energy varying as the inverse $2 / 5$ power of the number of beams. It is also set by the "Maschke current limit." In practical terms, for magnetic focusing, $I_{\max } \sim 4 \times 10^{5} B \beta^{2} a$ Amperes, where $B$ is the quadrupole field at the pole tip (T), $a$ the beam radius ( $\mathrm{cm}$ ), and $\beta$ the ratio of beam velocity to the speed of light (the expression for electric focusing is similar). Note that $I_{\max }$ varies as the beam radius, not the beam area. One maximizes the current through a given core by using a large number of small beams. Thus, the ion energy can be lowered (while still delivering enough total energy to the target) by neutralizing the beam in the chamber and/or using a large number of small beams. The ultimate limits are set by alignment and fabrication precision; a general prejudice against using many beams must be overcome by experimental experience.[5]

"Nominal" beam and pipe radii in an electrostatic-focusing accelerator have generally been assumed to be $a \sim 1.6 \mathrm{~cm}, b \sim 3 \mathrm{~cm}$. Recent experiments [16] have refined our understanding of quadrupole breakdown voltage as a function of size, and at present $a \sim 1 \mathrm{~cm}, b \sim 2.3 \mathrm{~cm}$ appears to be an optimal design point. (The calculated transportable current through a given pipe in a multi-beam array has not changed as a result of this new information, but the beams are smaller and there 
are more of them). Future improvements in technology may allow smaller apertures, with correspondingly larger multi-beam currents at the low-energy end.

\section{Recirculating Induction Accelerators}

A recirculating induction accelerator potentially offers reduced cost relative to a "conventional" linac because the accelerating and focusing elements are re-used many times in a single target shot. The overall accelerator length is reduced (to about $3.6 \mathrm{~km}$ in the "C-design" recirculator of Ref. [6], and possibly less), and the accelerating cores are smaller and are not driven so close to saturation because there is no need to accelerate the beam at the maximum possible rate. The recirculator designs considered to date employ greater axial compression than is typically assumed for linac designs, with a smaller number of longer beams used initially, and do not employ beam merging.

The beam dynamics issues which must be resolved before a recirculating driver can be built include centroid control, longitudinal control, avoidance of phase-space dilution in bends, and insertion/extraction of the beam into/out of the rings. As described in Subsection 6 below, these can be addressed at reduced scale in a small prototype recirculator. The waveform generators in a driver must supply variable accelerating pulses at high repetition frequencies of order $100 \mathrm{kHz}$, and accurate time-varying dipole fields with good energy recovery. These requirements are challenging, but advances in solid-state power electronics should make it possible to meet them through a technology development program; LLNL has already achieved $200 \mathrm{kHz}$ bursts at $5 \mathrm{kV}$ and $800 \mathrm{~A}$, with pulse widths of $0.5-2 \mu \mathrm{s}$, but with a non-variable format.[17] Because of its long path length, a recirculator driver will require a high vacuum of $\sim 10^{-10}$ to $10^{-11}$ torr. Collisional interactions (intrabeam charge exchange, ionization of background gas, stripping of beam ions by gas collisions) can drive beam or gas ions into the walls of the beam pipe, and so cause the desorption of material off the walls. This material will interact with the beam on its next pass. Thus, a high vacuum is especially important. There remain uncertainties in some of the relevant cross sections; many of these can be resolved through experiments on existing accelerator facilities.

Current research on recirculator drivers has centered on multi-ring designs, with each ring augmenting the beam's energy by an order of magnitude over 50 to 100 laps. Relative to a "conventional" linac, the length is reduced by a factor of order 23 , but the beam path length lengthened to perhaps $200 \mathrm{~km}$. Here too, the research program aims to develop the necessary physics and technology. Hybrid designs (with a recirculator at the low-energy end) are also possible.

\section{ILSE Program, Elise Accelerator, and ESQ Injector}

Early experiments with intense space-charge-dominated ion beams were very successful. These included large-aperture source development tests using cesium, as well as experiments performed on the SBTE and MBE-4 facilities at LBNL.[18,19] However, these experiments tested only a few driver elements: sources, injectors, beam matching, and acceleration with electric focusing. For economic reasons, the beams in SBTE and MBE-4 were small in comparison with those currently envisioned for a driver. 
To begin addressing the physics and technology issues associated with fullscale beams, LBNL, in collaboration with LLNL, formulated an accelerator project and suite of experiments known as ILSE (Induction Linac Systems Experiments). The full ILSE accelerator concept consisted of a four-beam electric-focusing accelerator bringing a potassium beam to $5 \mathrm{MeV}$, followed by a one-beam magnetic-focusing accelerator to $10 \mathrm{MeV}$.[8] The beam would have the same linecharge density as that of a driver, and will be of the same diameter. However, to reduce cost it would use a shorter pulse and lower energy. The critical dimensionless parameters relating betatron wavelengths to the accelerator lattice period would be the same as in a driver. Experiments to be performed as part of the ILSE sequence include beam merging, drift-compression and pulse-shaping, beam bending, focusing onto a spot, and recirculation.

At the request of the Department of Energy, a proposal for construction at LBNL of the four-beam electrostatic-focusing part of the ILSE accelerator (the elements up to about $5 \mathrm{MeV}$ ) was submitted. This electrostatic-focusing accelerator is known as "Elise."[7] In December of 1994, the Elise proposal received "Key Decision 1" approval, allowing engineering design to begin. However, funding for the U.S. HIF program has remained approximately level, and the $\Pi$ LSE/Elise project is currently "on hold."

In anticipation of the ILSE program of accelerator development and experiments, LBNL, in collaboration with LLNL, has developed, over the past two years, a single-beam injector which produces a full-scale beam suitable for injection into one channel of the ILSE/Elise accelerator. This injector is of a novel "ESQ" type, employing a sequence of electrostatic quadrupole lenses with a superposed voltage gradient along the axis. The net effect is to both confine and accelerate the beam. The system consists of a dished hot-plate source with an internal filament, an axisymmetric diode section taking the beam to $1 \mathrm{MeV}$, and the ESQ section. This section decouples the strict relationship between accelerating and focusing voltages inherent in earlier "electrostatic aperture column" designs, alleviates difficulties associated with breakdown limits, and produces a high-quality beam.[20].

\section{Planned Experiments in Bending and Recirculation}

A recirculating accelérator using the ILSE linac as its front end would represent an attractive facility. Before such an ILSE-scale recirculator can be credibly proposed, it will be necessary to develop the technology and physics on a smaller scale. Lawrence Livermore National Laboratory, in collaboration with Lawrence Berkeley National Laboratory, is currently developing a small prototype heavy-ion, intense beam, recirculating induction accelerator. This "small recirculator" is intended to explore, in a scaled manner, the physics and technology issues involved in constructing a full scale recirculating driver for application to inertial fusion energy. The small recirculator will be developed and operated as a series of experiments over several years' time. Figure 6 illustrates the overall design of the final small recirculator, and lists some of the elements which must all work together, both in it and in a full-scale fusion driver. 
The small recirculator will have a circumference of 14.4 meters, a $3.5 \mathrm{~cm}$ aperture radius (pipe radius) for the beam focusing and bending elements, and a half-lattice period of $36 \mathrm{~cm}$. The beam will be transversely focused with alternatinggradient permanent magnet quadrupoles with a field of $\sim 0.3 \mathrm{~T}$ at the pipe wall, and will be bent with electric dipole deflector plates. These quadrupoles and dipoles will each physically occupy about $30 \%$ of the axial lattice length, and the full recirculator ring will consist of 40 half-lattice periods, including several special large-aperture quadrupole magnets through which the beam will be inserted and extracted. The beam ion is singly charged potassium, and the beam will be accelerated from an initial particle kinetic energy of $80 \mathrm{keV}$ to $320 \mathrm{keV}$ over 15 laps by 34 induction cores. (No induction cores will be present in the lattice periods where the beam is

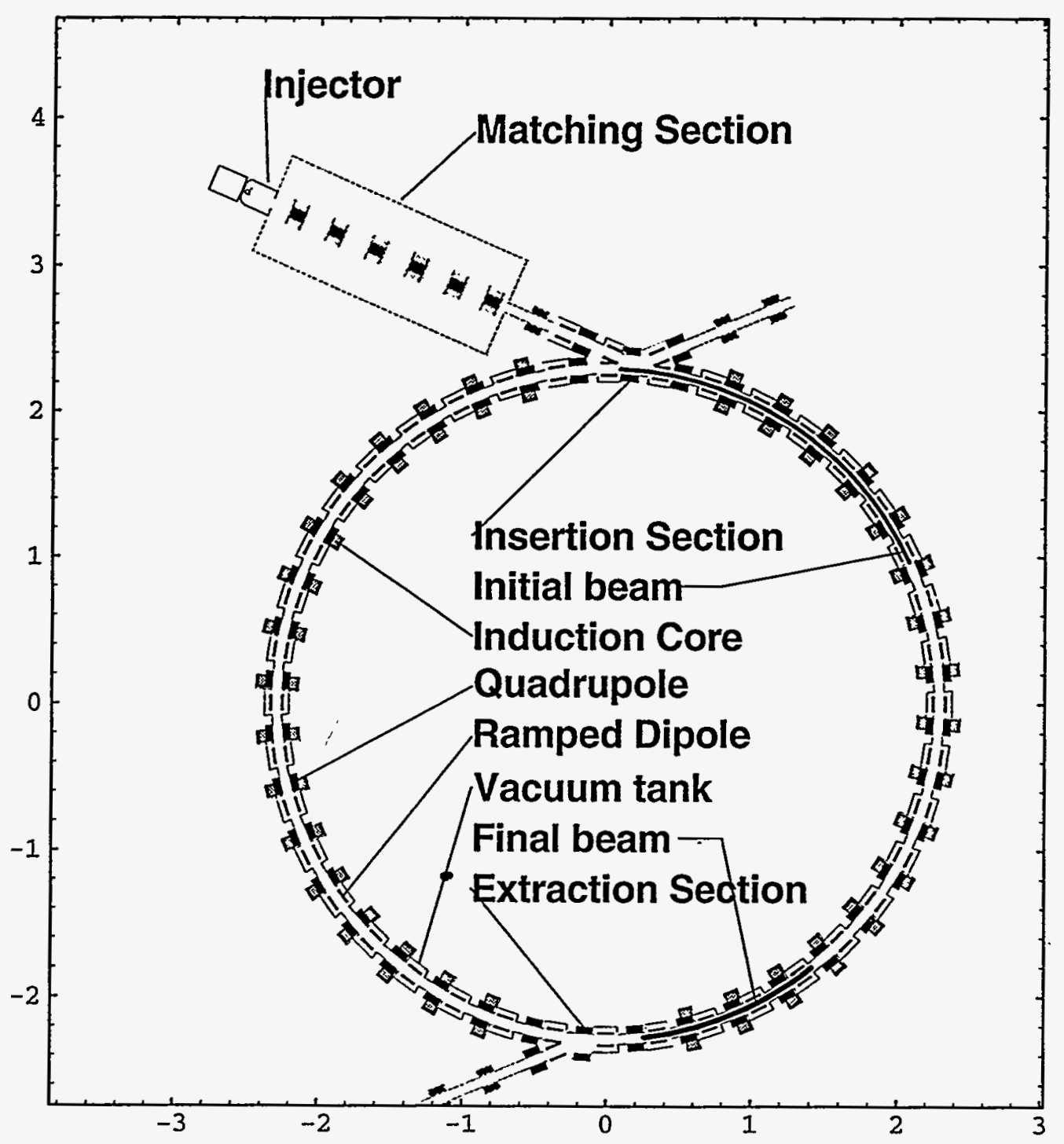

Fig. 6. Overview of final small recirculator configuration (scales in meters). 
inserted and extracted.) The initial current of the beam on insertion into the ring will be $2 \mathrm{~mA}$, corresponding to a line-charge density of $0.0036 \mu \mathrm{C} / \mathrm{m}$ and characteristic beam radius of $1.1 \mathrm{~cm}$, and the initial pulse duration will be $4 \mu \mathrm{sec}$. Also, the initial undepressed phase advance per lattice period will be $\sigma_{0}=78^{\circ}$, depressed by space charge to $\sigma=16^{\circ}$. After the full 15 laps of acceleration, the beam will have a final current of $8 \mathrm{~mA}$, with a corresponding line-charge density and average beam radius of $0.00721 \mu \mathrm{C} / \mathrm{m}$ and $1.3 \mathrm{~cm}$, and the final pulse duration will be $1 \mu \mathrm{sec}$. Also, at the final beam energy, the phase advances will decrease to $\sigma_{0}=45^{\circ}$ and $\sigma=12^{\circ}$ per lattice period.

Because the beam in the small recirculator will be nonrelativistic and accelerating, the waveforms required to accelerate and bend the beam will be technologically challenging. They will require the accurate synthesis of a train of $\sim 15$ detailed voltage pulses with a repetition rate ranging from approximately 40 to $90 \mathrm{KHz}$ at the initial and final beam energies. The voltage pulses for the electric bending dipoles must be correctly shaped and synchronized with respect to the pulses that power the induction cores. Furthermore, detailed "ear" pulse structures and lap-to-lap variation of the pulse duration must be added to the accelerating waveforms to maintain or decrease the beam length.

As of this writing, the source and injector diode are injecting a beam into the electrostatic-focusing matching section (a modified segment of LBNL's SBTE apparatus), which then injects the beam into a straight transport line. The matching section uses every second quadrupole of the original structure for focusing, with the quadrupole voltages individually tunable, and steering elements in some of the vacated quadrupole positions. The mechanical design of the recirculator "half-lattice period" is partially completed. Details of the experimental design $[9,21]$ and status $[9,22,23]$ were presented at the International Symposium, Princeton, 1995.

Linear experiments now under way are studying beam transport in a linear channel, using some of the quadrupole magnets destined for the recirculator. These experiments will afford phase-space measurements of the magnetic transport of space-charge-dominated ion beams. They will characterize the beam prior to injection into the recirculator, provide a test-bed for diagnostic development (especially the capacitive pickup centroid position monitors), and afford a preliminary assessment of the role of electrons in magnetic beam transport. Similar space-charge-dominated beam experiments using electromagnetic quadrupoles and longer pulses were carried out by the GSI group some years ago.[24]

Future experiments will study beam transport around a bend of order $90^{\circ}$ (at first without any accelerating modules). The transition of the beam from a straight transport line into the ring will represent a change of curvature, and will allow study of the resulting emittance growth. Emittance growth can also result from imperfections in the focusing and bending fields; the small imperfections expected in the experiments will be well characterized by theory and measurement. Even over a short bend, detailed intercepting beam diagnostics (using a two-slit apparatus to measure both transverse position and velocity) should be able to detect relatively small changes in the distribution of beam particles as a result of the bend. An important goal of these initial experiments will be validation of the computer models and scaling laws used to predict the behavior of linear and recirculating drivers. 
Later experiments will study insertion and extraction, acceleration, beam steering, bunch compression, and fully integrated operation of the recirculator. Until the ring is complete it will be possible to use intercepting diagnostics to characterize the beam and to calibrate the non intercepting diagnostics that will be critical to the successful operation of the full ring. As currently planned, the beam can be extracted and diagnosed with detailed intercepting diagnostics once or twice each lap. As with earlier linac experiments at LBNL, excellent shot-to-shot repeatability is anticipated and, so far, observed. The principal non intercepting diagnostic under development is a set of segmented capacitive pickups to be located inside the quadrupoles $[22,23]$.

Theoretical calculations clearly show that bend-induced emittance growth in driver-scale recirculators will be minimal.[25] The parameters of the small recirculator, with its sharp bends, are such that a measurable amount of emittance growth is expected to take place over the full fifteen laps, with most of this growth occurring in the first two laps, as confirmed by detailed 3-D particle-in-cell simulations.[26,27]

The long beam path length in the machine (up to, and possibly exceeding, 300 full lattice periods) will provide a unique opportunity to observe and characterize the longitudinal propagation of space-charge waves along the beam. Such waves will be launched by mismatching the applied ear fields. Also to be explored are issues important to both recirculating and linear drivers, such as slow thermalization.

\section{OTHER ELEMENTS OF THE U.S. HIF RESEARCH PROGRAM}

The U.S. HIF program is also carrying out an intensive program of technology development and smaller-scale experiments. In addition to the injector and recirculator efforts described above, and the technology development program now underway, three other expèrimental efforts are being pursued at LBNL.

The first of these is an experimental study of beam merging, using the MBE-4 apparatus with new, angled sources feeding into one of the existing transport lines through a new combining section.[15] The final element through which the four beams pass must both bend and focus the beams. Because there is no room for conventional electrostatic.quadrupole rods, the element is a "squirrel cage" made of individual wires, each held at the appropriate voltage. The beams are brought together in the "Stonehenge" configuration described above. The early stages of this experimental work have already had significant spin-off benefit in ion source improvements and understanding of beam current limits, which are proving larger than previously had been thought.

The second effort is a magnetic quadrupole development program. These "current dominated" quadrupoles are being designed with a geometry that will be suitable to the superconducting elements planned for a driver. Tests to date have focused on the mechanical and heat-transfer properties of the magnets; near-term tests will begin to consider their optical properties (the detailed winding design is still being optimized as of this writing). Experiments exploring beam transport through a set of such magnets will be carried out if funding is available, initially using the beam from LBNL's new ESQ injector.[28] 
The third is a study of plasma lenses, building on recent work in Europe. This work is being carried out in collaboration with A. Tauschwitz, a visitor at LBNL from GSI. Such lenses may be attractive as final optics, either with or without a channel through which the focused beam is transported to the target.[14]

A smaller experimental program at the University of Maryland [29] is studying longitudinal beam dynamics, including wave propagation, and most recently is using a channel with a resistive wall to explore the longitudinal microwave instability as it applies to HIF drivers, where it is driven by the impedance of the accelerating modules. Detailed theoretical [30] and computational [31,32] studies indicate that this instability will have a moderate growth rate, and that careful beam control (and possibly feed-forward stabilization techniques) will be necessary to preserve beam quality. The Maryland experiments will serve to validate and normalize these calculations.

Because the space-charge-dominated beams in an induction accelerator are effectively non-neutral plasmas, theoretical and computational modeling of these beams is carried out using techniques related to those used in both the accelerator and plasma physics communities. Models employed range from simple zerodimensional codes based upon analytically-derived scaling relations, through fluidand moment-equation simulations, up to large and elaborate discrete-particle simulations. In addition to LBNL and LLNL, theoretical and simulation efforts are underway at the Naval Research Laboratory [33], the Princeton Plasma Physics Laboratory [34], and MIT.[35]

The CIRCE code [36] is a multi-dimensional model which solves an envelope equation (evolving moments such as centroid position and transverse extent) for each of a number (typically a hundred or greater) of "slices" of the beam. The longitudinal dynamics of the beam is modeled by evolving the positions and velocities of the slices using fluid equations. CIRCE is used to assess alignment tolerances, accelerating schedules, and steering techniques in both linacs and recirculators. It is useful for any application in which the evolution of the detailed internal degrees of freedom of the beam need not be resolved (e.g., emittance growth processes); at present, the beam emittance is assumed constant in CIRCE.

Because the beam resides in the accelerator for relatively few plasma oscillation periods, particlé-in-cell simulation techniques are especially effective and have proved invaluable to the design and analysis of ongoing experiments, and to the prediction of the behavior of future machines. The WARP code includes both fully three-dimensional (WARP3d) [26,27] and axisymmetric (WARPrz) [31,32] particle-in-cell simulation models. The former is used for "near first-principles" studies of MBE-4, the ESQ injector, the small recirculator, the Elise accelerator, beam bending, beam combining, and other accelerator experiments and elements. The axisymmetric model is used for long-term beam dynamics studies including the effects of accelerating module impedance. WARP3d uses a number of novel techniques to render it both accurate and efficient. These include a capability for subgrid-scale placement of internal conductor boundaries, a capability of simulating "bent" accelerator structures, and a technique for rapidly following particles through a sequence of sharp-edged accelerator "lattice elements," using a relatively small number of time steps while preserving accuracy. On some problems (such as the ESQ injector), the code is run in a quasi-steady state mode, whereby a 3-D run can 
be completed in just a few minutes of computer time; this makes it suitable for iterative design calculations. The ultimate goal of this code development is effective simulations of both the ILSE experiments and a driver, from the source through the final focus, into the codes used to model propagation in the fusion chamber, and ultimately into the target design codes. A number of other particle-in-cell codes employ a "slice" description of the beam (assuming slow variation of quantities along the beam); much application, as well as detailed studies of the properties of such particle-in-cell models of beams and plasmas, has been carried out.[33]

In addition to these accelerator development efforts, the program is exploring all of the proposed modes of propagating the beams through the fusion chamber to the target. The BIC (Beam-in-Chamber) code [11,12] is an axisymmetric electromagnetic code which allows the computational grid to taper down to small radius, preserving resolution as the beam converges toward the target. It is used to examine the near-ballistic propagation modes, and such processes as beam neutralization and stripping. Higher-density pinched propagation modes are being studied at LBNL, at Sandia National Laboratory, and at the Naval Research Laboratory. [13]

LLNL is involved in target design research, using essentially the same tools developed for target experiments with the Nova and NIF lasers and the Sandia iondiode devices. The goal is to develop robust, high-gain targets which impose a minimal set of requirements on the driver. Recent progress has led to a much more detailed understanding of the radiation converters; integrated design efforts are well underway.[37]

Finally, research continues on improved HIF power-plant concepts. The most recent work, on an improved HYLIFE- $\Pi$ system with a liquid Flibe waterfall providing a renewable first wall, suggests an extremely competitive cost of electricity may be obtainable from an HIF power plant.[1] Recent studies of target injection and tracking [38], and planned experiments with model waterfalls [39], are important elements of the HIF power plant development program.

\section{SPACE-CHARGE-DOMINATED BEAM PHYSICS ISSUES}

The following "taxonomy" of issues was developed with induction-accelerator heavy-ion fusion in mind. It may have broader utility, but is not all-inclusive (even for HIF). The treatment is very brief, since much discussion might reasonably be devoted to each topic. No attempt is made to systematically cite all relevant references.

Note that, in general, U.S. HIF researchers generally use the term "spacecharge-dominated" (with various hyphenations!) to refer to a situation wherein the transverse force balance is primarily between the space charge and the applied focusing forces. Such beams are typically space-charge-dominated longitudinally as well. In contrast, European researchers often refer to beams as space-chargedominated when the longitudinal space charge force is greater the longitudinal thermal pressure, even when the beams are not transversely space-chargedominated. 


\section{Ideal beam "equilibria"}

\section{A. Utility of the concept}

Ideal equilibria have long been used as a tool for the analysis of transportable current, beam stability, etc. Since an accelerating beam in an alternating-gradient (A-G) lattice is not in true equilibrium, a question arises concerning the utility of approximate equilibrium as a goal for system design. Efforts to keep the beam "matched" to the transport channel minimize transverse particle excursions and keep more of the beam in the good-field region. Similarly, longitudinal matching minimizes the launching of waves on the beam. Thus, it is generally believed that beams which are "near equilibrium" will in general be well-behaved. However, it may be that in some cases the optimum design will involve a beam far from equilibrium, at some stage or stages of the acceleration process.

\section{B. Transverse equilibria for infinitely long beams}

For an axisymmetric system, or for a smooth-approximation treatment of an alternating-gradient system, the assumption of thermal equilibrium may be reasonable, especially if no time-varying external forces are applied. However, since the beams are practically collisionless, and instabilities etc. will in general not drive them all the way to thermal equilibrium, it is not clear that thermal states are reached on relevant time scales. An issue remains about the boundedness of thermal equilibria; in general they involve nonzero densities at infinity or on the pipe wall, and one must invoke exponential smallness of the error thus incurred. Furthermore, non-thermal equilibria might in principle be more appropriate in some cases.

For an $A-G$ system, one asks whether there exists a nonsingular (i.e., non Kapchinskij-Vladimirskij) 4-D (or 5-D if longitudinal velocity spread is included) phase space density describing a continuous beam which is replicated after one lattice period. Formally such existence requires absence of particle loss. It remains unclear how best to find such an equilibrium. In principle one could use the Vlasov equation to numerically evolve densities on a 4-D or 5-D mesh over one lattice period, then vary parameters (via, e.g., a quasi-Newton procedure) until a replicating state is found. In practice such a procedure would be extremely computationally intensive. Clever choice of a set of basis functions used to characterize the distribution may be the key to resolving this issue.

For one study of this area, see Ref. [46].

\section{Longitudinal equilibria at beam ends, assuming axisymmetry}

Questions to be resolved are: What are practical, achievable "ear" waveforms? What is the actual "equilibrium shape" of the beam tips (axial dependence of beam radius, line-charge density, emittance; ideally, the full 5-D particle distribution function), in the approximation that ear fields are continuously applied? Is there a particle distribution which replicates under periodic application of ear fields?

\section{Three-dimensional equilibria}

Little analytic work is likely to be done in full 3-D geometry. However, full 3-D simulations need appropriate initial conditions. In some cases the beam is best formed by simulating the injection process, but in others it is useful to start with an approximate equilibrium. In WARP simulations, specification of such initial states 
is usually carried out by assuming the beam has a semi-Gaussian distribution transversely (i.e., a top-hat function in position and a Gaussian in velocity), with a variety of longitudinal dependences. Often, the longitudinal velocity in the main, flat-top part of the pulse is assumed Gaussian, with the line-charge density flat over the main pulse and tapering to zero at the ends with (e.g.) a parabolic falloff. The transverse beam size in the tips is set to have a specified dependence upon the local line-charge density, often assumed (in an ad hoc manner) to preserve a constant charge density and depressed phase advance within the beam envelope. When such a beam is followed over many lattice periods, it is seen to evolve toward a blunter shape, with charge density decreasing toward the tips.

\section{Beams formed by injection}

\section{A. Beam ends}

Questions to be resolved include: What are the shapes (in the sense described above) of the beam head and tail as the beam exits the diode? What are the shapes downstream, at the first practical location for application of time-dependent accelerating fields? How best to "catch" or "patch" the beam as it emerges from the injector and encourage it toward equilibrium (or some other desired state)?

For discrete-particle simulations, efficient and accurate modeling of timedependent space-charge limited flow is a challenging problem, and the ideal algorithm has not yet (in this author's opinion) been identified.

\section{B. Transverse profile}

Questions to be resolved include: What is the transverse distribution function? What design strategy leads to a beam with minimal hollowing and/or distortion? What are the downstream effects of source nonuniformities?

\section{Longitudinal behavior}

Questions to be resolved include: What waves are launched from the beam ends as a result of the diode voltage rise and fall (for achievable rise and fall waveforms)? What waves are launched as a result of ripple or droop in the injection voltage? How do such wave motions thermalize?

As a result of acceleration, the longitudinal thermal velocity (in the beam frame) can be expected to decrease, in both the injector and the main accelerator (under certain assumptions).[40] However, effects such as rapid transfer of thermal energy from transverse to longitudinal motions (described below, in Subsection VI) mitigate the cooling. One must assess how much "accelerative cooling" actually occurs in the injection process for each circumstance, perhaps by detailed simulation, ideally by experiment.

\section{Transverse dynamics}

\section{A. Dynamic aperture in the presence of strong space charge}

Space charge introduces nonlinearities in the transverse fields both directly and through the effects of image charges. "Rules of thumb" have been established over the years for how much of the aperture can be used, avoiding the introduction of 
excessive nonlinear image effects and accounting for coherent oscillations. The aperture radius $b$ needed is often assumed to be given by $b=1.25 a+c$, where $a$ is the maximum beam radius and $c$ is an additive constant of order $1 \mathrm{~cm}$, to account for misalignments and beam nonuniformities (a smaller constant may be used at very low energy, before errors accumulate). Recent experimental investigations of breakdown limits for actual quadrupoles have established improved scaling laws for transportable current. [16] As pointed out by Laslett, for electrostatic quadrupole focusing systems using four round cylindrical rods per element, a rod:aperture radius ratio of 8:7 leads to cancellation of the unwanted dodecapole term, in the infinite-rod limit.[41] Other prescriptions take into account image effects.[42] This work should be updated. Also, progress in machining and aligning small elements suggests that future systems will optimize at a larger number of smaller beams passing in parallel through a given set of induction cores.

\section{B. Halo causes and avoidance}

Minimization of beam halo (stray particles) is of importance because of the desire to preserve the practicality of hands-on maintenance of the driver, while keeping the bore radius small enough that the driver cost is attractive. For spacecharge-dominated beams, the profile is closer to a "top hat" than to a Gaussian, and so (as in most astrophysical contexts) the halo particles constitute a distinct population rather than the tails of the main distribution.

Recent work on core-halo models has shown that a "breathing" core is capable of parametrically driving particles to large excursions and so into the halo. Minimization of mismatch is a clearly identified means of minimizing halo formation. Other measures involve avoidance of "hollow" beams and of beams with over-focusing at the edges, out of the injector. Longitudinal mismatches as well as waves launched by imperfect "ears" can in principle contribute to a transverse beam halo as well.

\section{Halo scraping}

In some contexts it may be attractive to periodically remove stray particles before they hit the wall in an unpredictable manner. The introduction of apertures at appropriate stations along the machine may effectively remove the halo. This also may ensure that the collisions with solid matter which occur when particles are lost are at near-normal incidence, thereby minimizing desorption from the wall (which is generally greater when an ion strikes at grazing incidence). In general it may be necessary to place multiple scrapers so that halo particles at all phases of their betatron oscillations are removed. The question of whether and how quickly the halo reforms must be answered in the context of each particular machine.

\section{Beam combining}

Transverse beam combining or merging, as described earlier, necessarily involves the entrainment of empty phase space. Much progress has been made in using simulations and experiments to understand the process, and further experiments are underway, but the optimum designs have not yet been identified. 


\section{E. "Recovery" of beam when perturbed}

After the beam has been perturbed by a change in system parameters leading to a mismatch, by scraping, or by some other process, it relaxes toward a new equilibrium. However, in the absence of collisions the relaxation occurs by other means and is typically incomplete. Many relaxation processes operate on the time scale of a plasma period, while others (such as those which damp centroid oscillations) can be much slower.

\section{F. Beam "steering" vs. precise alignment}

In a linear system it may be possible to precisely align all elements and only "steer" in a rudimentary way; however, true steering may afford simpler construction with relaxed tolerances and overall cost savings. Steering of the beam requires adjustment, on the basis of measurements of the beam centroid motion, of existing dipole fields, and/or of auxiliary fields. Such steering is essential to multilap operation of the small recirculator, and of driver-scale recirculators as well.

The principal method of beam steering would use beam centroid information obtained on the previous shot (linac), or on the corresponding lap of the previous shot (recirculator). Because of the expected good shot-to-shot repeatability of the system, this information should provide sufficiently reliable input to accomplish this task. This inter-shot (so-called "shot-to-shot") steering can most simply be carried out by setting the steering fields to a value which does not change as the beam passes by. Such "time-independent" steering should provide the bulk of the necessary correction.

In addition, it may be highly desirable to include a small amount of timedependent steering occasionally. This will serve to remove the small amount of accumulated centroid position error which arises from the head-to-tail energy and current variation of the beam.

The ultimate method of steering would rely on information from the current shot. The beam centroid position will be measured, and steering corrections applied at a location downstream from the measuring station. This "feed-forward steering" would have the advantage of removing random, non-repeatable errors, as well as those which remain systematic from shot to shot.

\section{G. Emittance growth in curved systems}

Emittance growth can result from the non-uniform distribution of beam space charge resulting from the action of centrifugal forces. One identified mechanism is a consequence of the beam's thermal longitudinal velocity spread; particles of differing energies naturally ride around the bend at differing radii, and the resulting transverse "smearing" of the space charge leads to nonlinear forces. As revealed in particle simulations using the WARP3d code[26] and confirmed by analytic theory,[25] the effect occurs at changes in the accelerator's curvature where the distribution of beam particles must relax toward a new equilibrium. Thus, a circular recirculator is to be preferred over one with a "racetrack" shape.

\section{H. Insertion and extraction into and out of rings}

In order to switch the beam into and out of a ring, it is necessary to apply dipole bending fields that vary in time. In a driver-scale recirculator, insertion and 
extraction are carried out over a number of half lattice periods. In the small recirculator it is possible to insert or extract the beam in a single half lattice period. However, even for the small ring, extraction will require real-time adjustment of the dipole immediately upstream of the extraction section as well as the dipole within that section. An additional requirement is that transverse confinement of the beam against its own defocusing space-charge forces must be carried out continuously, even during the insertion or extraction process, or else the beam would grow excessively in size. Furthermore, the optical quality of the magnetic quadrupole lenses which provide this transverse confinement must be high, or aberrations will distort the beam over multiple laps. To this end, a large-aperture quadrupole element must be employed; its inside dimension in the vertical plane need be no larger than that of a normal quadrupole, but its dimension in the plane of the ring must be larger by a factor of approximately 2.5 . For the small ring we plan to use a permanent-magnet quadrupole with an expanded aperture. For a driver, a set of special large-aperture electromagnets ("Panofsky quadrupoles") will be employed. Initially, the extraction process should be just a mirror-image of insertion; however, at higher energies extraction becomes more challenging because bending the beam becomes more difficult.

\section{Longitudinal dynamics}

\section{A. Wave propagation}

Waves if excited will propagate along the beam, with a restoring force associated with both space-charge and (usually to a lesser degree) thermal pressure. Much is known about the propagation of such waves, their mode structure and dispersion, both theoretically[30,31,32] and experimentally[29]. In some cases a "longitudinal instability" analogous to the microwave instability seen in storage rings is driven by the accelerating module impedance; recent work has elucidated the circumstances under which growth is significant.[32] Remaining questions concern the impedances of real accelerating structures, the utility of feed-forward stabilization, and the properties of various inherent stabilization mechanisms including longitudinal thermal spread and transverse-longitudinal interactions.

\section{B. Beam-end effects}

The shape and timing of the "ear" pulses is critical if the beam tips are to be quiescently confined. These are in general provided using separate waveform generators, and/or by shaping the initial rise of the accelerating pulses. Questions include: How to minimize the waves launched due to periodic application of ear fields? Can they be eliminated somehow? What are effects of various kinds of errors in ear fields: strength, timing, shape, etc.? How do waves reflect at bunch ends for confined, expanding beams?[29]

\section{Acceleration}

One issue is how best to shape the initial accelerating waveforms so as to perturb the beam minimally during early acceleration? One scenario is termed "loadand-fire," wherein no acceleration takes place until the entire pulse has been injected. Others include use of triangular ramps to start the pulse compressing, with 
a transition to flat-top pulses. In a short machine, optimum use of the available voltseconds is important.

A technology which needs to be fully explored is feedback correction of accelerating errors.

In a practical accelerator, tradeoffs between pulse compression, accelerative cooling (II.C), and "equilibration" (VI) must be taken into account

\section{Pulse compression $\&$ shaping}

In a driver, the beam current is increased (via a reduction of the pulse duration) by orders of magnitude. Some of this increase is due to the increase in the beam's velocity. The remainder is due to an imposed axial compression of the beam-it is made physically shorter-as it is accelerated. The design of the driver must take the pulse compression into account.

Current concepts assume that the beam will be compressed by an additional factor of 10-25 after it leaves the driver, in a "drift-compression" process, to achieve a shaped pulse with a duration of order $10 \mathrm{~ns}$ (for the main part). Optimization of this process, including an assessment of the relative merits of driven- vs. drift-compression, or of drift-compression with a "mid-course correction," remains to be done. Detailed scenarios for setting up the beam linecharge density and velocity for drift compression have yet to be worked out.

The ultimate limitations on the pulse shape are as yet unknown; this is an active area of study. While not all shapes are practical (and very short pulses are challenging), sufficient flexibility appears to be available that the needs of the fusion application can be met.

As the beam compresses axially its radial extent becomes larger. It thereby samples more of the non-linear fields from the accelerator lattice elements, creating more opportunity for emittance growth and possible beam loss. These effects must be taken into account.

\section{3-D dynamics}

3-D particle simulations of HIF-relevant beams are used to study effects introduced by the full geometry, and to do "integrated" simulations of full beams. Most beam physics can be successfully treated in lower dimensional approximation, but some cannot. For example, 4-D or 5-D "slice" models of infinite beams are often useful, but extra information is needed to know "what is in each slice" for real, finite-length beams with longitudinal particle motions in the beam frame. Axisymmetric 5-D models are useful but do not capture effects of A-G systems or of beam bending, not to mention merging and other strongly geometry-dependent processes.

\section{A. Transverse-Iongitudinal coupling in bent systems}

A bent system naturally couples the longitudinal and transverse in-plane motions. For example, in the small recirculator designed at LLNL, particles are slowed or sped longitudinally as they enter the region between the electrostatic dipole plates which serve to bend the beam, with a strong dependence upon their individual transverse position. This "energy effect" is analogous to the one calculated for and observed in the LBNL ESQ injector. If the whole beam centroid 
moves off the design orbit, the overall timing of the beam's passage around the ring is affected. Also, particles of differing energy naturally follow different orbits (III.G).

\section{B. Other dynamical coupling (due to fringe fields, etc.)}

Fringe fields and imperfections can also couple the various degrees of freedom. For example, particles at different transverse positions can be slowed or sped differently as they enter or exit a lattice element.

\section{Time-dependent offsets and "corkscrew"}

In a beam of non-constant energy, misalignments can lead to a time-dependent centroid displacement (or "corkscrew", as it appears in a system using solenoids). This is further complicated by space-charge image effects.

\section{BBU instability (nature and correction)}

The beam-breakup instability endemic to relativistic electron beams in induction accelerators is much milder in the context of HIF ion accelerators, but must still be kept in mind as a part of practical machine design.

\section{E. Halo formation due to imperfect beam end confinement}

Beam end confinement against electrostatic repulsive forces is effected by timedependent fields. Particles which happen to pass far into the tip before being driven back toward the main bunch may be imparted a large peculiar velocity, leading to poor matching and large transverse excursions.

\section{Equilibration and relaxation}

\section{A. Collective transfer of thermal energy from $x, y$ to $z$}

Simulations $[43,44]$ in both 3-D and $r, z$ geometry have shown the existence of a rapid collective transfer of thermal energy from transverse to longitudinal degrees of freedom, when the transverse temperature $T_{\perp}$ is significantly warmer than the longitudinal temperature $\mathrm{T}_{\| \mid}$. This appears to be a betatron-motion analog to the "Harris mode" in magnetoplasma, which is driven by cyclotron motion. In no instances have these simulations shown a similar transfer in the other direction, when $T_{\| l}>T_{\perp}$. An analytic theory [45] is under development. The nature of the seeds for growth, and the consequences for real systems design, need to be understood. The process may amount to a useful cooling mechanism for transverse motions, or may represent a constraint on fusion driver design. In injectors and elsewhere it may partially offset the "accelerative cooling" process described in (II.C).

\section{B. Existence or nonexistence of rapid transfer from $\mathrm{z}$ to $\mathrm{x}, \mathrm{y}$ ?}

Such a transfer might be induced by coupling due to fringe fields in external elements, or by the so-called "energy effect." It appears likely in short bunches, where the beam's fields provide a strong geometrical coupling.

\section{Existence of mechanisms to drive beam toward an equilibrium}

Mechanisms which drive the beam toward a quiescent state may be valuable, as noted in (I.A). Thermalization of mismatch oscillations typically brings with it 
emittance growth, but the beam may be well-centered and better-behaved as a result. Active cooling measures may be useful in some cases, though those identified to date are in general too slow for linac applications. Careful tailoring of accelerating and confining fields, possibly through a feedback or feed-forward process, can to some degree push the beam toward a state with quiescent macroscopic moments.

\section{CONCLUD́NG REMARKS}

The research program outlined here is, in the author's opinion, broadly based and well-positioned to explore the most important aspects of HIF driver physics and technology. It is complementary to the European program of RF driver development. At present no particular heavy-ion driver concept is clearly best. Each requires further development before its full potential can be assessed, and improvements to each appear both possible and likely. More work is needed integrating target designs, beam transport options, and driver optimization.

The categorization of beam dynamics issues outlined in the second part of this paper may provide a useful framework for future investigations.

The ongoing experimental program, in close conjunction with theory and modeling, will lead to confident predictions of driver behavior. The progression to full-scale beams and the requisite technology to accelerate and control them is a critical step in the development of heavy-ion fusion as an energy source.

\section{ACKNOWLEDGMENTS}

The work described in this paper is the result of a collaborative effort of the LBNL and LLNL HIF groups, along with colleagues at other institutions. The author thanks Roger Bangerter, John Barnard, Andy Faltens, Bill Herrmannsfeldt, and Grant Logan for useful comments on the manuscript.

*This work was performed under the auspices of the U.S. Department of Energy by LLNL under contract W-7405-ENG-48. 


\section{REFERENCES}

The most recent International Symposium on Heavy Ion Inertial Fusion was held at Princeton Plasma Physics Laboratory on September 6-9, 1995. The Proceedings of that meeting will be published in Fusion Engineering and Design (Elsevier, Amsterdam), in late 1996.

Il Nuovo Cimento 106 A, N. 11-12, (1994), Proceedings of the International Symposium on Heavy Ion Inertial Fusion, Frascati, Italy, May 25-28, 1993, is also a good general reference.

[1] R. W. Moir et. al., "HYLIFE-II: A Molten-Salt Inertial Fusion Energy Power Plant Design-Final Report," Fusion Technology 25, 5-25 (1994).

[2] W. R. Meier et. al., "OSIRIS and SOMBRERO Inertial Confinement Fusion Power Plant Designs Final Report," DOE/ER/54100-1 (1992).

[3] L. M. Waganer et. al., "Inertial Fusion Energy Reactor Design Studies Prometheus-L \& Prometheus-H," DOE/ER/54101 (1992).

[4] W. M. Sharp, "A Comparison of Driver Concepts for Heavy-Ion Fusion," Proceedings of the 11th Workshop on Laser Interaction and Related Plasma Phenomena, Monterey, CA, 25-29 October 1993, AIP Conference Proceedings 318, AIP Press (1994), G. Miley, Ed.; also ICF Quarterly Report, LLNL Report UCRL-LR-105821-94-2, Pp. 70-7 (1994).

[5] R. O. Bangerter, "The Induction Approach to Heavy-Ion Inertial Fusion: Accelerator and Target Considerations," Il Nuovo Cimento 106 A 11, 1445 (1994). [6] J. J Barnard, F. J. Deadrick, A. Friedman, D. P. Grote, L. V. Griffith, H. C. Kirbie, V. K. Neil, M. A. Newton, A. C. Paul, W. M. Sharp, H. D. Shay, R. O. Bangerter, A. Faltens, C. G. Fong, D. L. Judd, E. P. Lee, L. L. Reginato, S. S. Yu, and T. F Godlove, "Recirculating Induction Accelerators äs Drivers for Heavy-Ion Fusion," Phys. Fluids B 5, 2698 (1993).

[7] E. P. Lee, R. O. Bangerter, C. F. Chan, A. Faltens, J. Kwan, E. Henestroza, K. Hahn, and P. Seidl, "Physics Design and Scaling of Elise," Proc. Int. Sympos. on Heavy Ion Inertial Fusion, 1995.

[8] T. J. Fessenden et. al;, Plasma Phys. \& Cont. Nucl. Fus. Res. 3, 89 (1992).

[9] A. Friedman "Recirculating Induction Accelerators for Inertial Fusion: Prospects and Status," Proc. Int. Sympos. on Heavy Ion Inertial Fusion, 1995.

[10] D. D-M. Ho, I. Haber, K. R. Crandall, and S. T. Brandon, Part. Accel. 36, 141 (1991).

[11] D. A. Callahan and A. B. Langdon, "Transport of a Partially-Neutralized Ion Beam in a Heavy-Ion Fusion Reactor Chamber," Proc. 1995 IEEE/APS Part. Accel. Conf., Dallas TX, May 1995.

[12] D. A. Callahan, "Chamber Propagation Physics for Heavy Ion Fusion," Proc. Int. Sympos. on Heavy Ion Inertial Fusion, 1995.

[13] C. L. Olson et.al., "Physics of Gas Breakdown for Ion Beam Transport in Gas," Il Nuovo Cimento 106 A, N. 11, 1705 (1994)

[14] A. Tauschwitz, E. Boggasch, D. H. H. Hoffmann, M. de Magistris, U. Neuner, M. Stetter, R. Tkotz, T. Wagner, W. Seelig, and H. Wetzler, Il Nuovo Cimento 106 A, N. 11, 1733 (1994) 
[15] C. M. Celata, W. Chupp, A. Faltens, W. M. Fawley, W. Ghiorso, H. Hahn, E. Henestroza, C. Peters, and P. A. Seidl, "Transverse Combining of 4 Beams in MBE-4," Proc. 1995 Part. Accel. Conf., Dallas TX, May 1995

[16] P. Seidl and A. Faltens, "Electrostatic Quadrupoles for Heavy-Ion Fusion," Proc. 1993 Part. Accel. Conf., IEEE Cat. No. 93CH3279-7 p. 721 (1993).

[17] H. C. Kirbie, W. R. Cravey, S. A. Hawkins, M. A. Newton and C. W. Ollis, "A FET-Switched Induction Accelerator Cell," Proc. Ninth Int. Pulsed Power Conf., IEEE Cat. No. 93CH3350-6 p. 415 (1993)

[18] M. G. Tiefenback and D. Keefe, "Measurement of Stability Limits for a Space-Charge-Dominated Ion Beam in a Long A.G. Transport Channel," Proc. 1985 Part. Accel. Conf., IEEE Trans. Nucl. Sci. NS-32, p. 2483 (1985).

[19] T. Garvey, S. Eylon, T. J. Fessenden, and E. Henestroza, "Beam Acceleration Experiments on a Heavy Ion Linear Induction Accelerator," Part. Accel. 37-8, 241 (1992).

[20] S. S. Yu, "Driver-Scale Ion Injector Experiments," Proc. Int. Sympos. on Heavy Ion Inertial Fusion, 1995.

[21] J. J. Barnard, M. D. Cable, D. A. Callahan, T. J. Fessenden, A. Friedman, D. P. Grote, D. L. Judd, S. M. Lund, M. A. Newton, W. M. Sharp, and S.S. Yu, "Physics Design and Scaling of Recirculating Induction Accelerators: From Benchtop Prototypes to Drivers," Proc. Int. Sympos. on Heavy Ion Inertial Fusion, 1995.

[22] T. J. Fessenden, J. J. Barnard, M. D. Cable, F. J. Deadrick, M. B. Nelson, S. Eylon, and H. S. Hopkins, "Intense Heavy-Ion Beam Transport with Electric and Magnetic Quadrupoles," Proc. Int. Sympos. on Heavy Ion Inertial Fusion, 1995.

[23] F. J. Deadrick, J. J. Barnard, T. J. Fessenden, J. Meredith, and J. Rintamaki, "Development of Beam Position Monitors for Heavy Ion Recirculators," Proc. 1995 Part. Accel. Conf., Dallas, TX, May 1-5, 1995 (to be published).

[24] J. Klabunde et.al., IEEE Trans. Nucl. Sci NS-30 No. 4, 2543 (1983).

[25] J. J. Barnard, H. D. Shay, S. S. Yu, A. Friedman, and D. P. Grote, "Emittance Growth in Heavy-Ion Recirculators," Proc. 1992 Linear Accelerator Conf, August 24-28, Ottawa, Ontario, Canada, C.R. Hoffman, ed.; AECL 10728 (AECL Research, Chalk River, Canada) p. 229.

[26] A. Friedman, D. P. Grote, and I. Haber, "Three-Dimensional Particle Simulation of Heavy-Ion Fusion Beams," Phys. Fluids B 4, 2203 (1992)

[27] D. P. Grote, A. Friedman, I. Haber, and S. S. Yu, "Three-Dimensional Simulations of High-Current Beams in Induction Accelerators with WARP3d," Proc. Int. Sympos. on Heavy Ion Inertial Fusion, 1995.

[28] M. Stuart, A. Faltens, W. M. Fawley, C. Peters, and M. C. Vella, "Design and Construction of a Large Aperture, Quadrupole Electromagnet prototype for ILSE," Proc. 1995 IEEE/APS Part. Accel. Conf., Dallas TX, May 1995; see also W. M. Fawley, L. J. Laslett, C. M. Celata, A. Faltens, and I. Haber, "Simulation Studies of Space-Charge-Dominated Beam Transport in Large-Aperture Ratio Quadrupoles," Il Nuovo Cimento 106 A 11, 1637 (1994). 
[29] J. G. Wang, D. X. Wang, D. Kehne, M. Reiser, and H. Suk, "Studies of Space-Charge Waves due to Localized Perturbations in an Electron Beam," Il Nuovo Cimento 106 A, N. 11, 1745 (1994)

[30] E. P. Lee, "Longitudinal Instability in Heavy-Ion Fusion Induction Linacs," Il Nuovo Cimento 106 A, N. 11, 1679 (1994)

[31] D. A. Callahan, A. B. Langdon, A. Friedman, and I. Haber, "Longitudinal Beam Dynamics for Heavy-Ion Fusion," Proc. 1993 IEEE/APS Part. Accel. Conf., IEEE Cat. No. 93CH3279-7 p. 730 (1993).

[32] D. A. Callahan, "Simulations of Longitudinal Beam Dynamics of SpaceCharge Dominated Beams for Heavy Ion Fusion," Ph.D. thesis, University of CA, Davis, 1994; available as LLNL Report UCRL-LR-119364, Dec. 1994.

[33] I. Haber and H. Rudd, "Numerical Limits on P.I.C. Simulation of Low Emittance Transport," Proc. Conf. on Linear Accel. and Beam Optics Codes, La Jolla Inst., C. R. Eminhizer, Ed., AIP Conf. Proc. 177, AIP, NY, 1988, p. 161. [34] R. C. Davidson and Q. Qian, Phys. Plasmas 1, 1328 ff; 3104 ff (1994).

[35] C. Chen and R. C. Davidson, "Nonlinear Properties of the KapchinskijVladimirskij Equilibrium and Envelope Equation in a Periodic Focusing Field," Phys. Rev. E49, 5679 (1994).

[36] W. M. Sharp, J. J. Barnard, D. P. Grote, S. M. Lund, and S. S. Yu, "Envelope Model of Beam Transport in ILSE," Proc. 1993 Computational Accelerator Physics Conference, Pleasanton, CA, Feb. 22-26 1993, pp. 540-548. [37] D. D-M. Ho, J. A. Harte, and M. Tabak, "Radiation-Driven Targets for Heavy-Ion Fusion," Proc. 15th Int. Conf. on Plasma Phys. and Controlled Nucl. Fusion Research, IAEA, Seville, Sept. 26-Oct. 1, 1994 (IAEA-CN-60-B-P-13).

[38] R. Petzoldt, "Inertial Fusion Energy Target Injection, Tracking, and Beam Pointing," Ph.D. thesis, University of CA, Davis, March 7, 1995; available as LLNL Report UCRL-LR-120192.

[39] K. B. Wilson and P. Peterson, "HYLIFE-II Inertial Confinement Fusion Reactor Oscillating Sheet Jet Experiment Design," Master of Science Project report, U.C. Berkeley Dept. of Nuclear Engineering, 1995 (unpubl.); M. Longeot and P. Peterson, abstract sub. to 12th Topical Meeting on the Technology of Fusion Energy, June 16-20, 1996, Reno NV, proc. to be publ. in Fusion Technology.

[40] M. Reiser, Theory, and Design of Charged Particle Beams, Wiley, New York, 1994, p. $393 \mathrm{ff}$.

[41] L. J. Laslett, in Selected Works of L. Jackson Laslett, Lawrence Berkeley Laboratory Pub-616, V. III, chap. 6 (1987).

[42] C. M. Celata, Proc. 1987 Part. Accel. Conf., p. 996 (1987).

[43] A. Friedman, D. A. Callahan, D. P. Grote, A. B. Langdon and I. Haber, Bull. Am. Phys. Soc. 35, 9, 2121 (1990).

[44] I. Haber, D. A. Callahan, A. Friedman, D. P. Grote, and A. B. Langdon, "Transverse-Longitudinal Energy Equilibration in a Long Uniform Beam," Proc. 1995 Part. Accel. Conf.

[45] S. M. Lund, private communication.

[46] J. Struckmeier and I. Hofmann, "The Problem of Self-Consistent Particle Phase Space Distributions for Periodic Focusing Channels," Part. Accel. 39, 219249 (1992) 

